\title{
Sex differences in sensitivity to electric shock in rats and hamsters
}

\author{
WILLIAM W. BEATTY and RICHARD G. FESSLER \\ North Dakota State University, Fargo, North Dakota 58102
}

\begin{abstract}
Shock thresholds were measured in rats and hamsters of both sexes using both the flinch-jump and spatial-preference techniques. In agreement with earlier work, female rats had lower thresholds in the flinch-jump test, but there was no sex difference when sensitivity was assessed by the spatial preference method. Hamsters displayed a different pattern of sex differences. Female hamsters had higher aversion thresholds in the spatial preference test, but there was no sex difference in the flinch-jump test.
\end{abstract}

Previous research has demonstrated that female rats are more sensitive to electric shock than males. Qualitatively similar sex differences have been observed using flinch-jump (e.g., Beatty \& Beatty, 1970; Beatty \& Fessler, 1976; Marks \& Hobbs, 1972) or spatialpreference (Paré, 1969) techniques to measure shock thresholds, and females also perform instrumental responses to terminate shock more rapidly than males (Beatty \& Beatty, 1970; Davis, Porter, Burton, \& Levine, 1976).

The present experiment was designed to compare sensitivity to electric shock in rats and hamsters under comparable conditions. The hamster is an interesting organism for comparative studies of sex differences in behavior because on many of the physiological and behavioral measures that differentiate the sexes in most mammalian species sex differences are absent or reversed in hamsters (e.g., Gaskin \& Kitay, 1970; Lawlor, 1963; Marques \& Valenstein, 1976; Zucker, Wade, \& Zeigler, 1972).

\section{METHOD}

\section{Subjects}

The subjects were 20 male and 20 female albino rats obtained from the Holtzman Company, Madison, Wisconsin, and 21 male and 20 female golden hamsters (Mesocricetus auratus) obtained from ARS-Sprague-Dawley, Madison, Wisconsin. The animals were approximately 10 weeks old when they arrived in the laboratory. Upon arrival, they were caged singly in an air conditioned animal room with free access to Purina Lab Chow pellets and water. Lights in the animal room were on from 0900 to 2100 . Half of the animals of each sex and species were assigned randomly to groups that were tested in the flinchjump or spatial-preference tasks. Testing began 2 weeks after arrival in the lab and was conducted during the afternoon.

\section{Apparatus}

Flinch-jump tests were conducted in clear plastic chambers that contained grid floors made of bronze welding rod. For rats, the testing chamber measured $23.5 \times 25.0 \times 23.0 \mathrm{~cm}$ high. For hamsters, a similar box that measured $15.2 \times 15.3 \times$ $15.0 \mathrm{~cm}$ high was used. Spatial-preference tests were conducted in short runways made of clear plastic with floors of bronze

Supported by Grant HD 08206 from NICHHD. welding rod. The apparatus used for rats measured $60.0 \mathrm{x}$ $10.2 \times 13.8 \mathrm{~cm}$ high. For hamsters, a similar chamber that measured $36.2 \times 7.7 \times 9.4 \mathrm{~cm}$ high was employed. Photocells located near the midpoint of the apparatus monitored the location of the subject controlling timers and counters that recorded performance. Scrambled ac shock was delivered from a Grason-Stadler E700 source. This device can deliver a wide range of presettable current intensities. The values $.05, .06$, $.08, .10, .13, .16, .20, .25, .30, .40, .50, .60, .80,1.0,1.3,1.6$, and $2.0 \mathrm{~mA}$ were used in the present work. In the flinch-jump tests, white noise masked the sound of the shocker which was located in a room adjacent to the room containing the animal. For the spatial-preference tests the shocker, programming, and recording equipment were also located in an adjacent room.

\section{Procedure}

Flinch-jump tests. Pilot work quickly revealed that our standard procedure of presenting alternating ascending and descending series of shocks in a single session (see Beatty \& Beatty, 1970) was poorly suited to hamsters, because after they received a few strong shocks they maintained an erect posture, usually with the forepaws placed against the wall, for protracted periods. We abandoned this procedure because it was not possible to maintain reasonably comparable intershock intervals. Additional pilot work indicated that the problem described above could be largely circumvented by presenting only a single series of shocks in ascending order each test day. The first shock given was a $.05 \mathrm{~mA}$ for $.5 \mathrm{sec}$. As soon as the animal again assumed a posture with all four feet on the grid, another shock of higher intensity was presented for the same duration. An observer, located in an adjacent room, controlled presentation of the shocks and classified behavior into four mutually exclusive categories: no response, flinch (any detectable movement induced by the shock), shuffle (flinch followed by movement of the feet, usually at shock offset), or jump (all four feet leave the grid in response to the shock). Test trials on a given day continued with progressively increasing shocks until the animal jumped on two consecutive shock presentations. We defined the shock threshold for each response as the current required to elicit a particular response reliably (i.e., twice in a row). In both species the various behaviors typically appeared in the order listed above as the current was increased. Hence, if the animal exhibited a response normally seen at low intensity followed by a response usually observed at higher intensity (e.g., a flinch followed by a shuffle), then the threshold recorded (the flinch threshold in this example) was the current that elicited the normally more "activated" behavior (shuffle in the above example). In this manner separate thresholds were established for each response for each subject on each of the 5 test days. Results reported are based on the average threshold for each response and each subject over the five tests. 


\section{Spatial Preference}

Testing began when the animal was placed in the shock side of the apparatus. Time spent on the shock and nonshock sides as well as the number of shuttling responses made were recorded during a 5-min test session. On the following day, the current was increased one step and the positions of the shock and nonshock sides were reversed. Testing continued in this fashion until the animal spent $90 \%$ of its time on the nonshock side on two consecutive tests or was tested twice at a current of $.4 \mathrm{~mA}$. The aversion threshold was defined as the current value at which the animal spent $75 \%$ or more of its time on the nonshock side for two consecutive tests. If no value could be determined by this method, the value $.4 \mathrm{~mA}$ was assigned.

\section{RESULTS AND DISCUSSION}

Table 1 presents performance on the several threshold measures. As expected from previous work, female rats had lower thresholds on the flinch, shuffle, and jump measures. The changes in testing procedures and definition of the thresholds resulted in numerically higher values for these thresholds than we have reported in our earlier work (e.g., Beatty \& Beatty, 1970; Beatty $\&$ Fessler, 1976), but the sex difference in sensitivity to shock in this test was quite clear despite the minor procedural differences.

Paré (1969) reported that female rats had lower aversion thresholds than males using the spatial preference technique to measure the aversion threshold, but we did not observe a sex difference in the present experiment. It must be emphasized that the present findings in no sense constitute a failure to replicate Parés results, because of the many procedural differences between the two studies. The most important of these appears to be the fact that Pare exposed each of his animals to one (and only one) current intensity and manipulated current intensity as a between-subjects variable, while we exposed each of our animals to an ascending series of different current intensities. This latter procedure was beset by a serious problem: Several of the rats (6 of 20) quickly developed clear side preferences and spent nearly all of their time on one side of the apparatus, regardless of which side was shocked. These animals were easily recognized by their relatively low levels of shuttling and their persistent attraction to one side of the chamber in the face of increasingly intense shocks, so we reexamined the data excluding performance of animals that developed spatial preferences. Again no sex difference could be detected. In other studies we have tried to prevent development of side preferences by shortening the testing sequence, using only currents near the expected threshold, em-

Table 1

Mean Thresholds (mA)

\begin{tabular}{lcccc}
\hline & Flinch & Shuffle & Jump & $\begin{array}{c}75 \% \\
\text { Aversion }\end{array}$ \\
\hline Male Rats & $.17^{*}$ & $.51^{*}$ & $1.15^{*}$ & .26 \\
Female Rats & .14 & .42 & .86 & .24 \\
Male Hamsters & .11 & .26 & 1.11 & $.21^{*}$ \\
Female Hamsters & .12 & .25 & .92 & .27 \\
\hline
\end{tabular}

${ }^{*} p<.01$ for sex difference by $t$ test. ploying different test chambers, or switching the spatial position of the shock every minute within the session. In every instance, several rats developed side preferences. At present, there does not seem to be a satisfactory and economic way to measure aversion thresholds in the rat with the spatial-preference method. Our technique is clearly unsatisfactory, and while Paré's method presumably avoids the problems of our procedure, it does so at enormous cost in terms of the number of subjects required to determine the threshold.

The pattern of sex differences in hamsters was quite different from that observed in rats. There was no sex difference on any of the measures obtained in the flinch-jump tests, but female hamsters had reliably higher aversion thresholds in the spatial-preference test. In contrast to the problems encountered using the latter technique with rats, none of the hamsters developed side preferences.

The present findings support the general conclusion of a number of recent experiments (e.g., Lawlor, 1963; Marques \& Valenstein, 1976; Zucker et al., 1972) that have compared the behavior of male and female hamsters under conditions where male and female rats behave in a qualitatively or quantitatively different fashion. Clearly, the hamster is not a rat and theorists who would attempt to develop mechanisms to explain sex differences in human behavior based on experiments with rats might take note of this simple fact.

\section{REFERENCES}

Beatty, W. W., \& Beatty, P. A. Hormonal determinants of sex differences in avoidance behavior and reactivity to electric shock in the rat. Journal of Comparative and Physiological Psychology, 1970, 73, 446-455.

BeATTY, W. W., \& Fessler, R. G. Ontogeny of sex differences in open field behavior and sensitivity to electric shock. Physiology and Behavior, 1976, 16, 413-417.

Davis, H., Porter, J. W., Burton, J., \& Levine, S. Sex and strain differences in leverpress shock escape behavior. Physiological Psychology, 1976, 4, 351-356.

Gaskin, J. H., \& KitaY, J. I. Adrenocortical function and the hamster: Sex differences and effects of gonadal hormones. Endocrinology, 1970, 87, 779-786.

LAWLOR, M. Social dominance in the golden hamster. Bulletin of the British Psychological Society, 1963, 16, 1-14.

Marks, H. E., \& Hobbs, S. H. Changes in stimulus reactivity following gonadectomy in male and female rats of different ages. Physiology and Behavior, 1972, 8, 1113-1119.

Marques, D. M., \& Valenstein, E. S. Another hamster paradox: More males carry pups and fewer kill and cannibalize young than do females. Journal of Comparative and Physiological Psychology, 1976, 90, 653-657.

PARÉ, W. P. Age, sex and strain differences in the aversive threshold to grid shock in the rat. Journal of Comparative and Physiological Psychology, 1969, 69, 214-218.

Zucker, I., WAde, G. N., \& Zeigler, R. Sexual and hormonal influences on eating, taste preferences, and body weight of hamsters. Physiology and Behavior, 1972, 8, 101-111.

(Received for publication April 18, 1977.) 